\title{
Los ejemplos en la enseñanza de las ecuaciones en libros de texto de Educación
}

\section{Primaria}

\section{Os exemplos ao ensinar as equações em livros didáticos de Ensino Fundamental}

\author{
Margarita Cárcamo \\ Colegio Emprender, Osorno, Chile \\ margarita.carcamo@gmail.com \\ Danilo Díaz-Levicoy \\ Universidad de Granada (UGR), Granada, España \\ dddiaz01@hotmail.com \\ Cristian Ferrada \\ Universidad de Granada (UGR), Granada, España \\ adarref@hotmail.com
}

\section{Informações do Artigo}

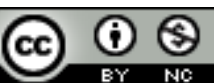

Histórico do Artigo

Submissão:

15 de novembro de 2017 .

Aceite:

26 de junho de 2018.

Palabras clave

Ejemplos

Ecuaciones

Libros de Texto

Educación Primaria

\section{Resumen}

En el presente trabajo mostramos resultados preliminares de un estudio sobre los ejemplos que se presentan en libros de texto de Educación Primaria para la enseñanza de las ecuaciones en Chile. Para tal efecto se realiza un análisis de contenido en los textos de $1^{\circ}$ a $6^{\circ}$ de matemática, que entregó el Ministerio de Educación durante el año escolar 2017. El análisis ha mostrado que las ecuaciones, y ejemplos de cómo trabajarlas, están presentes en los seis cursos de Educación Primaria, pese a que las directrices curriculares no las explicitan. Las variables utilizadas en la investigación corresponden a las representaciones utilizadas, los tipos de enunciados y los ejes temáticos en que aparecen. Los resultados permiten observar que mayoritariamente: las representaciones usadas en los ejemplos son conjuntamente las pictóricas y simbólicas; los enunciados usados son de tipo conceptual; y se encuentran en el eje de Números y operaciones, Patrones y álgebra. Además, se observa la ausencia de ejemplos sobre ecuaciones relacionados con el eje de datos y probabilidades, y de forma escasa los relativos a medición y geometría, donde deberían tener un mayor protagonismo las ecuaciones y ejemplos de cómo trabajarlas.

\section{Resumo}

Palavras-chave

Exemplos

Equações

Livros Didáticos

Ensino Fundamental
O presente trabalho mostra os resultados preliminares de um estudo sobre os exemplos que são apresentados nos livros didáticos dos anos iniciais do Ensino Fundamental para o ensino de equações no Chile. Para isso, uma análise de conteúdo foi realizada em livros didáticos do $1^{\mathrm{a}}$ ao $6^{\mathrm{a}}$ ano de matemática disponibilizados pelo Ministério da Educação durante 2017. A análise mostrou que as equações e exemplos de como trabalhálas estão presentes nos seis anos deste nível de ensino mesmo que as diretrizes curriculares não as especifiquem. As variáveis utilizadas na pesquisa correspondem às representações utilizadas, aos tipos de enunciados e os eixos temáticos em que aparecem. Os resultados nos permitem observar principalmente que: as representações usadas conjuntamente nos exemplos são pictóricas e simbólicas; os enunciados utilizados são conceituais; e elas são apresentados nos eixos de Números e operações, Padrões e álgebra. Além disso, observamos a ausência de exemplos sobre equações relacionados ao eixo de dados e probabilidades (estatística e probabilidade), e de forma escassa as relacionadas a medições e geometria, onde as equações e exemplos de como trabalhá-las devem ter maior proeminência. 


\section{Introducción}

En esta investigación abordamos tres aspectos importantes en la enseñanza de la matemática. En primer lugar, están las ecuaciones, un objeto matemático que está presente en todos los niveles educativos y que son fundamentales para entender y aplicar otros temas (CASTRO et al., 2017; GODINO; FONT, 2003; HERNÁNDEZ; ANDONEGUÍ, 2003; SANTIBÁÑEZ et al., 2009), pero que lamentablemente se trabaja de forma algorítmica (OLFOS, 2006). En segundo lugar, tenemos los libros de texto, uno de los recursos pedagógicos de mayor tradición y prestigio al interior de las salas de clases (BRAGA; BELVER, 2016; GÜEMES, 1994; SILVA, 1996). Y, en tercer lugar, los ejemplos, que se usan en la de enseñanza y aprendizaje de un tema, como una herramienta para explicar ciertos procesos (DÍAZ-LEVICOY et al., 2015; GOLDENBERG; MASON, 2008; WATSON; MASON, 2005). En nuestro caso son los ejemplos usados en los libros de texto, y que entendemos como aquella parte de él, en que se explican, aclararan o definen ideas o conceptos que el estudiante debe aprender (DÍAZ-LEVICOY et al., 2015).

Basado en estas tres consideraciones es que, en este trabajo, nos planteamos como objetivo analizar los ejemplos sobre ecuaciones que se utilizan en los libros de texto de Educación Primaria en Chile, y de esta forma caracterizar cómo se enseña este tema, por medio de los ejemplos, entregando información que puede ser considerada por profesores en la organización del proceso de instrucción.

En lo que sigue describimos los fundamentos de nuestro estudio (importancia de las ecuaciones, su rol en la enseñanza de la matemática y la importancia de los libros de texto), para seguir con la descripción de la metodología, los resultados obtenidos y, finalmente las conclusiones.

\section{Fundamentos}

\subsection{Importancia de las ecuaciones}

El estudio del álgebra es planteado como una generalización de la aritmética, pues utiliza las nociones y los enfoques que se usan en ésta última (KIERAN; FILLOY-YAGUE, 1989). Sin embargo, como señalan Kieran y Filloy-Yague (1989, p. 229), "el álgebra requiere un cambio en el pensamiento del estudiante de las situaciones numéricas concretas a proposiciones más generales sobre números y operaciones". El paso de la aritmética al álgebra exige un dominio tanto de las expresiones aritméticas, como del lenguaje algebraico, y de la utilización de simbología propia del álgebra, reconociendo en ello una dificultad para su aprendizaje (HERNÁNDEZ; ANDONEGUí, 2003). Las dificultades en el aprendizaje del álgebra son un tema de interés investigativo, y en las que Kieran y Filloy-Yague (1989) mencionan la asimilación de la estructura de las expresiones algebraicas, en particular, influyen en el trabajo con las ecuaciones. 
Castro et al. (2017, p. 164) evidenciaron otras dificultades, tales como "el uso del signo igual, problemas de palabras, solución de ecuaciones lineales, equivalencia de expresiones algebraicas, modelación, entre otros". En ambas investigaciones se resalta la importancia de las tareas algebraicas, que se deben trabajar para la introducción temprana de este tipo de razonamiento, pues es difícil comprender que "los estudiantes lleguen con años de 'experiencia' en los distintos tratamientos y maneras de entender y concebir las ecuaciones y que hayan logrado consolidar muy poco de este objeto matemático al terminar sus estudios" (HURTADO, 2013, p. 1046).

Por otro lado, autores como Lins y Gimenez (2001) mencionan que unir la enseñanza de la aritmética y el álgebra es un desafío en la comunidad científica, ya que éstas tienen aspectos en común. Aunque la separación está dada por la naturaleza del contenido matemático y el desarrollo cognitivo del estudiante (CARRAHER et al., 2006).

En el trabajo algebraico, las ecuaciones tienen un rol importante. Las ecuaciones de primer grado con una incógnita se entienden como "igualdades que solo se verifican o son verdaderas para un determinado valor numérico" (BERNAL, 2011, p. 4). En el mismo sentido, Rojas (2010, p. 122) señala que "una ecuación comprende tanto de los términos dados y las operaciones de las expresiones a ambos lados de la igualdad, como la relación de igualdad y sus propiedades".

En el contexto de la Educación Primaria chilena, las bases curriculares orientan en la formación de conceptos abstractos, utilizando permanentemente "la progresión de lo concreto a lo pictórico (icónico) y a lo simbólico (abstracto) en ambos sentidos, lo que se denomina con la sigla COPISI" (MINEDUC, 2012, p. 87).

Las ecuaciones tienen su justificación en el eje de Patrones y álgebra del currículo de Educación Primaria, el que menciona:

\begin{abstract}
En este eje se pretende que los estudiantes expliquen y describan relaciones de todo tipo, como parte del estudio de la matemática. Los estudiantes buscarán relaciones entre números, formas, objetos y conceptos, lo que los facultará para investigar las formas, las cantidades y el cambio de una cantidad en relación con otra. Los patrones (observables en secuencias de objetos, imágenes o números que presentan regularidades) pueden ser representados en forma concreta, pictórica y simbólica, y los estudiantes deben ser capaces de transportarlos de una forma de representación a otra, extenderlos, usarlos y crearlos. La percepción de los patrones les permite predecir y también fundamentar su razonamiento al momento de resolver problemas. Una base sólida en patrones facilita el desarrollo de un pensamiento matemático más abstracto en los niveles superiores, como es el pensamiento algebraico (MINEDUC, 2012, p. 91).
\end{abstract}

Sin embargo, el trabajo con ecuaciones se puede encontrar de manera implícita en los otros ejes del currículo (Números y operaciones, Geometría, Medición, y Datos y probabilidades), ya que mediante ellas se desarrolla del pensamiento algebraico, y diferentes habilidades de carácter transversal.

Al estudiar el tratamiento de las ecuaciones en el currículo de Educación Primaria vemos que éstas se especifican explícitamente desde el tercer curso, manteniéndose hasta el sexto curso (Cuadro1), presentando un progreso gradual, desde aspectos más sencillos a otros más complejos (KIERAN; FILLOY, 1989). 
Cuadro 1 - Las ecuaciones en el currículo de Educación Primaria.

\begin{tabular}{|c|c|c|}
\hline Curso & Objetivo de aprendizaje & Indicadores de evaluación \\
\hline $\begin{array}{l}\text { Tercero de } \\
\text { Educación } \\
\text { Primaria } \\
\text { (MINEDUC, } \\
\text { 2013a, p. 86) }\end{array}$ & $\begin{array}{l}\text { Resolver ecuaciones de un } \\
\text { paso, que involucren } \\
\text { adiciones y sustracciones y } \\
\text { un símbolo geométrico que } \\
\text { represente un número } \\
\text { desconocido, en forma } \\
\text { pictórica y simbólica del } 0 \\
\text { al } 100 \text {. }\end{array}$ & $\begin{array}{c}\text { Describen y explican una operación inversa con ayuda } \\
\text { de las relaciones numéricas en una "familia de } \\
\text { operaciones", por ejemplo, } 6,7 \text { y } 13 \text { en forma concreta, } \\
\text { pictórica y simbólica: } \\
6+7=13 \text { a } 7+6=13 \\
13-7=6 \text { a } 13-6=7 \\
\text { Resuelven una ecuación, aplicando estrategias como: } \\
\text { ensayo y error } \\
\text { "utilizar la operación inversa" en forma concreta, } \\
\text { pictórica y simbólica. }\end{array}$ \\
\hline $\begin{array}{l}\text { Cuarto de } \\
\text { Educación } \\
\text { Primaria } \\
\text { (MINEDUC, } \\
\text { 2013b, p. 101) }\end{array}$ & $\begin{array}{l}\text { Resolver ecuaciones e } \\
\text { inecuaciones de un paso, } \\
\text { que involucren adiciones y } \\
\text { sustracciones, } \\
\text { comprobando los } \\
\text { resultados en forma } \\
\text { pictórica y simbólica del } 0 \\
\text { al 100, aplicando las } \\
\text { relaciones inversas entre la } \\
\text { adición y la sustracción. } \\
\end{array}$ & $\begin{array}{c}\text { Modelan ecuaciones con una balanza, real o } \\
\text { pictóricamente; por ejemplo: } x+2=4 \text {. } \\
\text { Modelan inecuaciones con una balanza real que se } \\
\text { encuentra en desequilibrio; por ejemplo: } 2+x<7 \text {. } \\
\text { Modelan ecuaciones e inecuaciones de un paso, } \\
\text { concreta o pictóricamente, con una balanza y además } \\
\text { con software educativo. } \\
\text { Resuelven adivinanzas de números que involucran } \\
\text { adiciones y sustracciones. }\end{array}$ \\
\hline $\begin{array}{l}\text { Quinto de } \\
\text { Educación } \\
\text { Primaria } \\
\text { (MINEDUC, } \\
\text { 2013c, p. 58) }\end{array}$ & $\begin{array}{l}\text { Resolver problemas, } \\
\text { usando ecuaciones de un } \\
\text { paso que involucren } \\
\text { adiciones y sustracciones, } \\
\text { en forma pictórica y } \\
\text { simbólica. }\end{array}$ & $\begin{array}{c}\text { Expresan un problema mediante una ecuación donde la } \\
\text { incógnita está representada por una letra. } \\
\text { Crean un problema para una ecuación dada. } \\
\text { Obtienen ecuaciones de situaciones imaginadas sin } \\
\text { resolver la ecuación. } \\
\text { Resuelven una ecuación simple de primer grado con } \\
\text { una incógnita que involucre adiciones y sustracciones. } \\
\text { Evalúan la solución obtenida de un problema en } \\
\text { términos del enunciado del problema. } \\
\text { Explican estrategias para resolver problemas, utilizando } \\
\text { ecuaciones. }\end{array}$ \\
\hline $\begin{array}{l}\text { Sexto de } \\
\text { Educación } \\
\text { Primaria } \\
\text { (MINEDUC, } \\
\text { 2013d, p. 82-83) }\end{array}$ & $\begin{array}{l}\text { Representar } \\
\text { generalizaciones de } \\
\text { relaciones entre números } \\
\text { naturales, usando } \\
\text { expresiones con letras y } \\
\text { ecuaciones. }\end{array}$ & $\begin{array}{c}\text { Escriben y explican la fórmula para encontrar el } \\
\text { perímetro de un rectángulo. } \\
\text { Escriben y explican la fórmula para encontrar el área de } \\
\text { un rectángulo. } \\
\text { Usan letras para generalizar la propiedad conmutativa } \\
\text { de la adición y la multiplicación. } \\
\text { Describen la relación entre los valores en una tabla, } \\
\text { usando una expresión en que intervienen letras. } \\
\text { Representan la regla de un patrón, usando una } \\
\text { expresión en que intervienen letras. }\end{array}$ \\
\hline
\end{tabular}

Fuente: Directrices curriculares (MINEDUC, 2013a, b, c y d).

\subsection{Los ejemplos en la enseñanza de la matemática}

Los estudios realizados al uso de ejemplos son relevantes, ya que "influyen en el aprendizaje de los estudiantes" (CÁRCAMO, 2017, p. 5). Razón por la cual se convierten en un objeto de interés para este estudio. El concepto de ejemplo señala que es una palabra utilizada en variados aspectos: ilustraciones de conceptos y principios, definiciones generales y teoremas, técnicas específicas, ejercicios, situaciones para generar patrones, contextos para motivar la matemática, entre otros (WATSON; MASON, 2005). En el mismo sentido, Goldenberg y Mason (2008) manifiestan que pueden ser vistos como herramientas culturales mediadoras entre los alumnos y los conceptos matemáticos, los teoremas y las técnicas. Permitiendo la relación entre 
las ideas abstractas y la comunicación matemática, en forma individual o colectiva, proporcionando contexto, ayudando a distinguir características esenciales de un tema o contenido matemático. Rowland (2008) indica que el uso de ejemplos es una práctica pedagógica habitual para incorporar conceptos abstractos y caracterizar procedimientos generales. Por su parte, Watson y Chick (2010) los definen como instantes específicos de trabajo, desde algo particular para llegar a algo general. Los ejemplos en la enseñanza de la matemática se visualizan en diversos recursos: pizarra, libros de texto, guías de aprendizaje, entre otros (ZODIK; ZASLAVSKY, 2008). En el estudio de Otero et al. (2000) observaron dos tipos de ejemplos presentes en los libros de texto: los no resueltos y los resueltos, de estos últimos se destaca la importancia que tienen como guía para el estudiante, y manifiestan que por lo general estos son sencillos, para otorgar una comprensión inicial del tema.

De manera especial, en esta investigación, se trabajarán los ejemplos utilizados en los libros de texto, los cuales lo entenderemos como "una sección [...] que se utiliza para aclarar o definir algún concepto o idea, así como para mostrar al estudiante un algoritmo o procedimiento" (DÍAZ-LEVICOY et al., 2015, p. 737).

\subsection{Importancia de los libros de texto}

Los libros de texto son motivos de diferentes estudios, dado el papel fundamental y de tradición en el proceso de enseñanza y aprendizaje de diferentes disciplinas (SILVA, 1996) y que se han ido adaptando al desarrollo tecnológico (BRAGA; BELVER, 2016).

Son considerados un ejemplo de transposición didáctica (CHEVALLARD, 1991), ya que en él se presenta una adaptación de los contenidos de manera que sean enseñados y aprendidos por estudiantes de un determinado curso. Se espera que estos sean una implementación de las directrices curriculares (DÍAZ-LEVICOY; ROA, 2014; HERBEL, 2007; SHIELD; DOLE, 2013), influyendo significativamente en el éxito de su implementación (CANTORAL et al., 2015; GÜEMES, 1994). Vidal (2010) menciona que uno de los criterios para evaluar la calidad de los libros de texto está dado por el cumplimiento de las directrices curriculares. Para Alsina (2000) el libro de texto es un recurso que se corresponde con un nivel intermedio entre el currículo oficial y el currículo impartido, denominando currículo potencial, es decir, se refiere a aquello susceptible a ser trabajado en el aula.

El libro de texto ayuda y facilita la labor del profesor en la planificación, y desarrollo de sus clases, ya que como indica Güemes (1994, p. 34):

en el texto se encuentra la metodología que posibilita el desarrollo de los objetivos, se presentan ya seleccionados y secuenciados los contenidos (con sus definiciones, ejemplos, interrelaciones, etc.), se propone un banco de actividades sobre los mismos, se encuentra implícita la estrategia de enseñanza que ha de seguir el profesor en la presentación de la información, e incluso (a través de la guía didáctica o del profesor) se ofrecen algunas pruebas de evaluación del aprendizaje. 
Pese a esta relevancia, es necesario que el profesor vigile epistémicamente con contenidos con el fin de evitar errores que puedan ser asimilados por los estudiantes (ORTIZ, 2002).

\section{Metodología}

En esta investigación seguimos una metodología cualitativa (PÉREZ-SERRANO, 1994), de nivel descriptivo (HERNÁNDEZ et al., 2006) y mediante análisis de contenido (LÓPEZNOGUERO, 2002). La muestra estuvo formada por los seis libros de texto que ha entregado el MINEDUC a las escuelas y colegios municipales y particular subvencionados en el año académico 2017 (Ver detalle en Cuadro 2). En cada libro de texto hemos identificado los ejemplos que estén relacionados con las ecuaciones y analizado las siguientes variables:

1. Representaciones. En ella consideramos las notaciones pictóricas, simbólicas o gráficas, con las cuales se expresan conceptos, características o propiedades de los ejemplos (CÁRCAMO, 2017). Visualizadas como una habilidad dentro de las directrices curriculares de matemática en Educación Primaria y que, según Rowland (2008), cada ejemplo posee una representación respecto a cómo se desea utilizar. Janvier (1987) se refiere a que es una estrategia esencial para el aprendizaje de la matemática abstracta, permitiendo al estudiante observar diversidad, e ideas matemáticas más cercanas. Con esta variable observaremos si existe o no diversidad de formas de representación, dado que una compresión adecuada de las ecuaciones, por medio de los ejemplos, debe considerar el equilibrio de diferentes representaciones.

2. Tipo de enunciado. Esto consiste en identificar forma de los textos, verbales o conceptuales, con los que se comunican los ejemplos (CÁRCAMO, 2017). Enunciados verbales que usualmente son utilizados en la enseñanza del álgebra como una traducción del lenguaje cotidiano al algebraico y viceversa que a veces "dificulta a los estudiantes a plantear la ecuación que la represente" (BERNAL, 2011, p. 4). Los enunciados conceptuales que abordan "los términos dados y las operaciones de las expresiones a ambos lados de la igualdad, como la relación de igualdad y sus propiedades" (ROJAS, 2010 , p. 122). Con esta variable identificamos los aspectos que reciben mayor importancia en los ejemplos de las ecuaciones en libros de texto, y de esta forma ver qué se debe reforzar al momento de desarrollar la instrucción.

3. Eje de aprendizaje. Consiste en identificar el área de la matemática que se está trabajando en el libro de texto en particular, con el contenido de ecuaciones. EI MINEDUC (2012) establece que en Educación Primaria se trabajan los siguientes ejes que guían en proceso de enseñanza y aprendizaje: Números y operaciones; Patrones y álgebra; Geometría; Medición; y Datos y probabilidades. Con esta variable pretendemos visualizar si los ejemplos en que intervienen las ecuaciones, se utilizan en todos los ejes de aprendizaje, 
confirmando o no lo transversal de este tema.

Cuadro 2 - Libros de texto usados en el estudio.

\begin{tabular}{|c|c|c|c|}
\hline Curso & Autores & Autores & Editorial \\
\hline $1^{\circ}$ & Cortés (2017) & $\begin{array}{l}\text { Texto del Estudiante. } \\
\text { Matemática } 1^{\circ} \text { Básico }\end{array}$ & Cal y Canto \\
\hline $2^{\circ}$ & $\begin{array}{l}\text { Ho Kheong, Ramakrishnan, } \\
\text { Pui Wah y Choo (2017) }\end{array}$ & $\begin{array}{l}\text { Mi Matemática. Texto del } \\
\text { Estudiante } 2^{\circ}\end{array}$ & Marshall Cavendish Education \\
\hline $3^{\circ}$ & Charles et al. (2014) & $\begin{array}{c}\text { Matemática } 3^{\circ} \text { Educación } \\
\text { Básica } \\
\end{array}$ & Pearson \\
\hline $4^{\circ}$ & Andrews et al. (2014a) & Matemática $4^{\circ}$ Básico & Galileo \\
\hline $5^{\circ}$ & Andrews et al. (2014b) & Matemática $5^{\circ}$ Básico & Galileo \\
\hline $6^{\circ}$ & Andrews et al. (2014c) & Matemática $6^{\circ}$ Básico & Galileo \\
\hline
\end{tabular}

Fuente: Elaborado por los investigadores.

\section{Resultados}

Luego del análisis de los libros de texto oficiales que distribuye el MINEDUC a las escuelas y colegios durante el año escolar 2017. En la Tabla 1 vemos que la mayor cantidad de ejemplos relacionados con ecuaciones están entre $2^{\circ}$ y $4^{\circ}$ de Educación Primaria, donde se concentra el $67,7 \%$ de ellos, destacando en $2^{\circ}$ curso con un $29 \%$, pese a que el trabajo con las ecuaciones solo se especifica a partir de $3^{\circ}$ de primaria. Además, observamos ejemplos sobre ecuaciones en todos los niveles considerados en el estudio, confirmando la importancia de este tema para la formación matemática de los escolares.

Tabla 1 - Distribución de los ejemplos analizados.

\begin{tabular}{ccc} 
Nivel & Frecuencia & Porcentaje \\
\hline $1^{\circ}$ & 4 & 6,5 \\
$2^{\circ}$ & 18 & 29 \\
$3^{\circ}$ & 13 & 21 \\
$4^{\circ}$ & 11 & 17,7 \\
$5^{\circ}$ & 7 & 11,3 \\
$6^{\circ}$ & 9 & 14,5 \\
\hline Total & 62 & 100
\end{tabular}

Fuente: Elaborado por los investigadores.

\subsection{Representaciones}

La primera variable considerada en este estudio fueron las representaciones usadas en los ejemplos, es decir, nos fijamos en elementos usados para describir la actividad (notaciones pictóricas, simbólicas o gráficas). En el estudio hemos encontrado dos tipos de representaciones y que se mencionan a continuación. 


\section{CONOZCO}

llustración 1 - Ejemplo de representación pictórica y simbólica.

Para equilibrar una balanza debe haber igual cantidad de elementos en ambos lados, para ello puedes:

- Agregar elementos iguales:

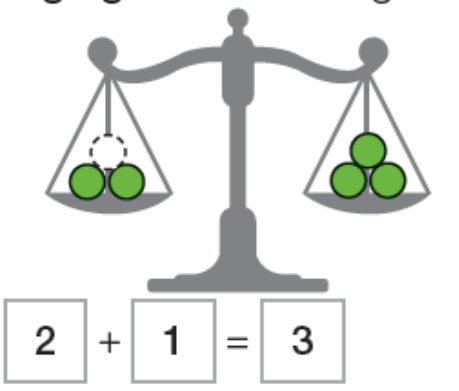

- Quitar elementos iguales:

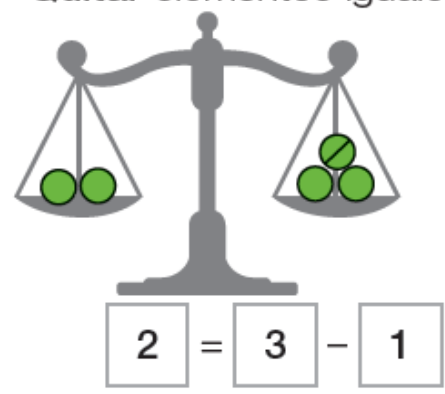

Fuente: Cortés (2017, p. 159).

En la llustración 1vemos un ejemplo donde se trabaja la forma de equilibrar una balanza, por medio de agregar y quitar elementos iguales, óptima para los años iniciales de Educación Primaria. En tal caso se usan representaciones simbólicas y pictóricas. Lo pictórico está asociado al uso de la balanza con esferas, y lo simbólico asociados a las igualdades que están bajo de cada una de las balanzas y que representan el agregar o quitar esferas.

Un segundo tipo de representación que observamos en los ejemplos relacionados con ecuaciones son las simbólicas, tal como vemos en la llustración 2. En tal ejemplo vemos una situación en que se tienen 7 ramos con 5 flores cada uno y se pide encontrar en total. La operación de multiplicación usada se expresa en forma de ecuación $(7 \cdot 5=$ ?), solución a la que se llega, contando de cinco en cinco.

llustración 2 - Ejemplo de representación simbólica.

\section{Ejemplo:}

Florencia tiene 7 ramos de flores.

Cada ramo tiene 5 flores.

¿Cuántas flores tiene en total?

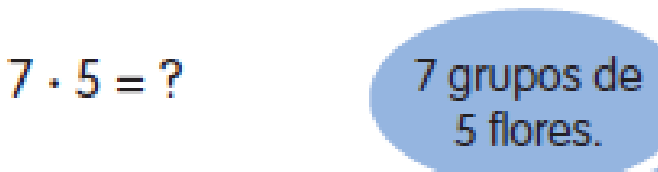

Florencia tiene 35 flores en total.
Yo cuento de cinco en cinco. $5,10,15,20,25$, 30,35

Fuente: Ho Kheong et al. (2017, p. 156). 
La Tabla 2 muestra la distribución de los ejemplos según las representaciones que se usan en ellos, donde en todos los casos se usan las representaciones simbólicas y en algunos casos combinados con pictóricas.

En esta tabla observamos, a nivel general, que la combinación de representaciones simbólicas y pictóricas son las que predominan en los ejemplos de los libros de texto $(64,5 \%)$. Tan solo en dos de niveles ( $4^{\circ}$ y $5^{\circ}$ de Educación Primaria) son en los que predomina exclusivamente la representación simbólica. En el segundo curso es donde está la mayoría de ejemplos con representaciones simbólicas y pictóricas $(88,9 \%)$, mientras que en quinto está el mayor porcentaje en que se usa la representación simbólica $(71,4 \%)$.

Tabla 2 - Distribución de los ejemplos según la representación usada.

\begin{tabular}{|c|c|c|c|c|c|c|c|c|c|c|c|c|c|c|}
\hline \multirow{2}{*}{ Representación } & \multicolumn{2}{|c|}{$1^{\circ}$} & \multicolumn{2}{|c|}{$2^{\circ}$} & \multicolumn{2}{|c|}{$3^{\circ}$} & \multicolumn{2}{|c|}{$4^{\circ}$} & \multicolumn{2}{|c|}{$5^{\circ}$} & \multicolumn{2}{|c|}{$6^{\circ}$} & \multicolumn{2}{|c|}{ Total } \\
\hline & $\mathbf{F}$ & $\mathbf{P}$ & $\mathbf{F}$ & $\mathbf{P}$ & $\mathbf{F}$ & $\mathbf{P}$ & $\mathbf{F}$ & $\mathbf{P}$ & $\mathbf{F}$ & $\mathbf{P}$ & $\mathbf{F}$ & $\mathbf{P}$ & $\mathbf{F}$ & $\mathbf{P}$ \\
\hline Simbólica & 1 & 25 & 2 & 11,1 & 4 & 30,8 & 6 & 54,5 & 5 & 71,4 & 4 & 44,4 & 22 & 35,5 \\
\hline Simbólica y pictórica & 3 & 75 & 16 & 88,9 & 9 & 69,2 & 5 & 45,5 & 2 & 28,6 & 5 & 55,6 & 40 & 64,5 \\
\hline Total & 4 & 100 & 18 & 100 & 13 & 100 & 11 & 100 & 7 & 100 & 9 & 100 & 62 & 100 \\
\hline
\end{tabular}

\subsection{Enunciado}

La segunda variable en estudio son los enunciados en los que se presentan los ejemplos. En nuestro caso hemos encontrado dos tipos de enunciados, los verbales y los conceptuales. Un ejemplo clasificado dentro de los enunciados verbales lo vemos en la llustración 3, donde el ejemplo formula una actividad y lo soluciona por medio del planteamiento de una ecuación, extraída del lenguaje verbal, explicando paso a paso cómo se ha solucionado.

Ilustración 3 - Ejemplo con enunciado verbal.

1. Hay muchos equipos de voluntarios para alimentar a los leones en la Reserva León. Otra reserva necesita ayuda, por lo tanto 10 equipos de voluntarios se van. Al día siguiente, 4 nuevos equipos de voluntarios llegan, y ahora la Reserva León tiene 15 equipos. ¿Cuántos equipos había originalmente?

Primero, elige una variable. Señala lo que la variable representa.

Después, escribe una ecuación.

Luego, trabaja desde el final hasta el principio.

Por último, resuelve la ecuación.
Usa $v$ para representar el número de equipos de voluntarios.

$$
\begin{aligned}
& v-10+4=15 \\
& 15-4+10=v \\
& 21=v
\end{aligned}
$$

Fuente: Andrews et al. (2014a, p. 168). 
En la llustración 4 se muestra un ejemplo con un enunciado conceptual, ya que está basado en el uso de símbolos matemáticos, relacionados con el cálculo mental de una suma. En este caso concreto se plantea una igualdad, donde el lado izquierdo está formado por la suma de 17 y 42, mientras que el lado derecho tiene el resultado (59) destacado con otra fuente (la misma con la que se debiesen hacer los cálculos mentales).

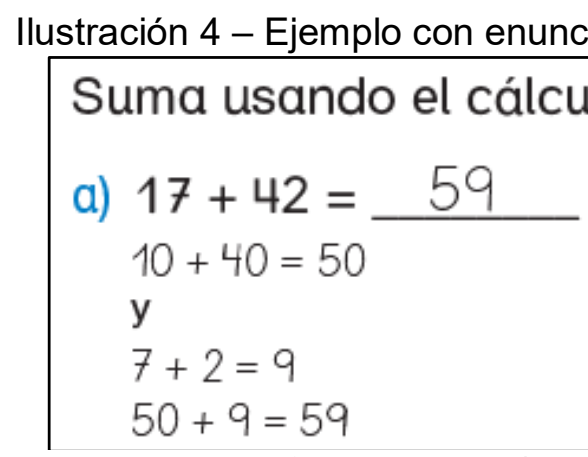

Fuente: Charles et al. (2014, p. 52).

En la Tabla 3 vemos la distribución de los ejemplos según el tipo de enunciado que usan. A nivel general, al igual que en cuatro de seis cursos, el tipo de enunciado con mayor presencia en los ejemplos con ecuaciones es el conceptual $(58,1 \%)$, en los que es posible observar paso a paso la solución de ecuaciones. El enunciado de tipo verbal predomina en $4^{\circ}$ y $6^{\circ}$ de Educación Primaria, con un $72,7 \%$ y $66,7 \%$, respectivamente.

Tabla 3 - Distribución de los ejemplos según el tipo de enunciado.

\begin{tabular}{|c|c|c|c|c|c|c|c|c|c|c|c|c|c|c|}
\hline \multirow{2}{*}{ Enunciado } & \multicolumn{2}{|c|}{$1^{\circ}$} & \multicolumn{2}{|c|}{$2^{\circ}$} & \multicolumn{2}{|c|}{$3^{\circ}$} & \multicolumn{2}{|c|}{$4^{\circ}$} & \multicolumn{2}{|c|}{$5^{\circ}$} & \multicolumn{2}{|c|}{$6^{\circ}$} & \multicolumn{2}{|c|}{ Total } \\
\hline & $\mathbf{F}$ & $\mathbf{P}$ & $\mathbf{F}$ & $\mathbf{P}$ & $\mathbf{F}$ & $\mathbf{P}$ & $\mathbf{F}$ & $\mathbf{P}$ & $\mathbf{F}$ & $\mathbf{P}$ & $\mathbf{F}$ & $\mathbf{P}$ & $\mathbf{F}$ & $\mathbf{P}$ \\
\hline Verbal & 0 & 0 & 6 & 33,3 & 5 & 38,5 & 8 & 72,7 & 1 & 14,3 & 6 & 66,7 & 26 & 41,9 \\
\hline Conceptual & 4 & 100 & 12 & 66,7 & 8 & 61,5 & 3 & 27,3 & 6 & 85,7 & 3 & 33,3 & 36 & 58,1 \\
\hline Total & 4 & 100 & 18 & 100 & 13 & 100 & 11 & 100 & 7 & 100 & 9 & 100 & 62 & 100 \\
\hline
\end{tabular}

\subsection{Ejes de aprendizaje}

En tercer lugar, analizamos el eje al que pertenecen las unidades en que hemos encontrado los ejemplos. De los cinco ejes mencionados en el currículo chileno de matemática para la Educación Primaria, solo hemos observado su presencia en cuatro de ellos.

En la llustración 5 vemos un ejemplo relacionado con el eje de Números y operaciones. En él se plantea una ecuación sencilla $(8+7=$ ?) y donde la solución, se sugiere, por medio de la descomposición numérica. 
Ilustración 5 - Ejemplo en el eje Números y operaciones.

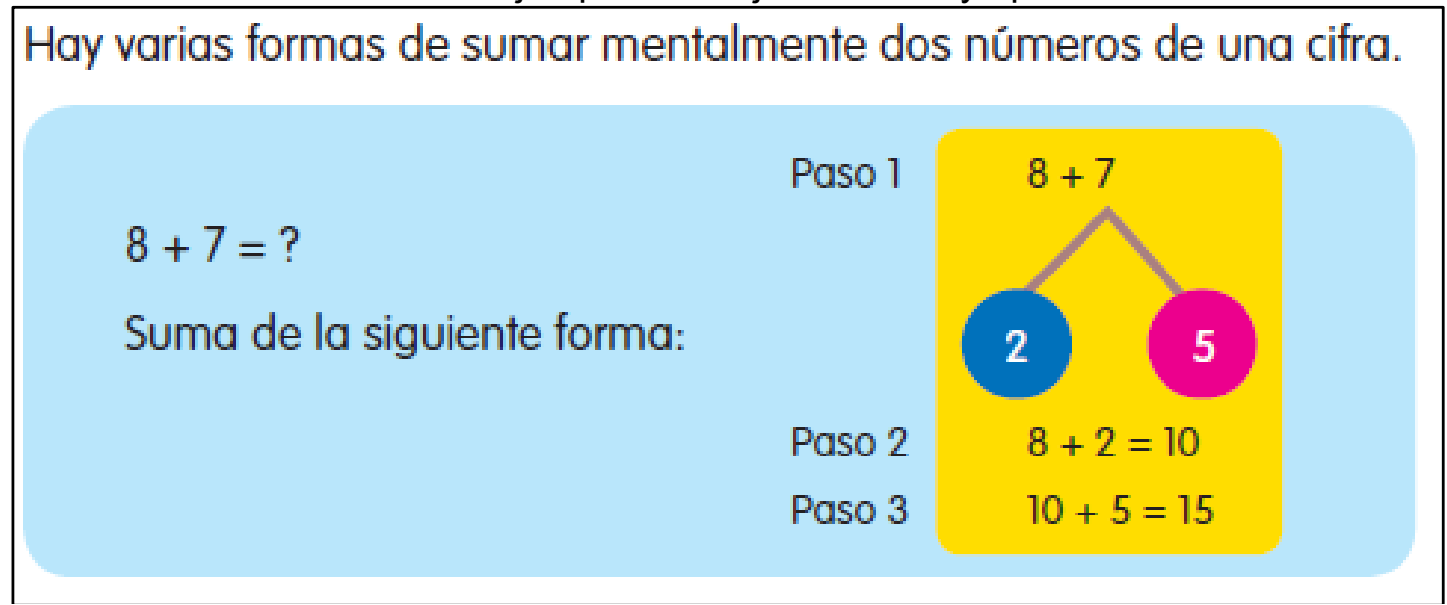

Fuente: Ho Kheong et al. (2017, p. 49).

Uno de los ejemplos que hemos encontrado en el eje de Patrones y álgebra lo vemos en la Ilustración 6 , donde se explica cómo solucionar una ecuación sumando el inverso aditivo de 2 en ambos lados de la igualad para encontrar el valor de la incógnita. Creemos importante que este tipo de situaciones sean trabajadas en clases, ya que va más allá de aplicar el algoritmo "pasa con el signo contrario" y el estudiante puede ver con claridad de dónde vienen estos procesos. Además, de esta forma los estudiantes trabajan los axiomas y propiedades de los números reales.

Ilustración 6 - Ejemplo en el eje Patrones y álgebra.

Utiliza las propiedades de las igualdades.
\[ \begin{aligned} y-2 & =13 \\ y-2+2 & =13+2 \quad \text { Sumas 2 a ambos lados. } \\ y & =15\end{aligned} \]

Fuente: Andrews et al. (2014b, p. 264).

En la llustración 7 vemos el ejemplo que se encontró en una de las unidades relacionadas a los temas de geometría. Dicho ejemplo hace referencia a la relación entre caras, vértices y aristas en prismas y pirámides, según los lados de la base. Luego, se presenta un cubo y una pirámide de base cuadrada en los que se comprueban las cantidades de caras, vértices y aristas. 
Ilustración 7 - Ejemplo en el eje Geometría.

\begin{tabular}{|c|c|}
\hline $\begin{array}{l}\text { Descubrió que el número de caras, vé } \\
\text { están relacionados. }\end{array}$ & rtices y aristas en pris \\
\hline $\begin{array}{l}\quad \text { Prismas } \\
\text { lados }=\text { número de lados en la base } \\
\text { lados }+2=\text { caras } \\
\text { lados } \cdot 2 \text { = vértices } \\
\text { lados } \cdot 3=\text { aristas }\end{array}$ & $\begin{array}{l}\quad \text { Pirámides } \\
\text { lados }=\text { número de lados en la base } \\
\text { lados }+1=\text { caras } \\
\text { lados }+1=\text { vértices } \\
\text { lados } \cdot 2=\text { aristas }\end{array}$ \\
\hline
\end{tabular}

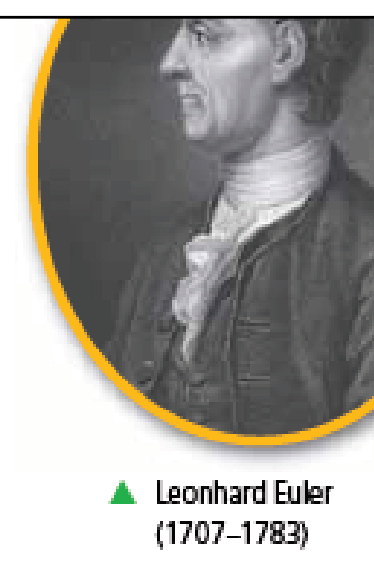

\section{Ejemplos}

\section{A Encuentra el número de caras,} vértices $\mathrm{y}$ aristas de un cubo.

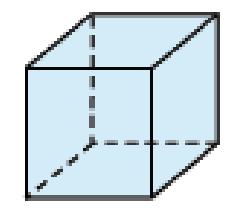

Un cubo tiene 4 lados en la base.

$$
\begin{aligned}
& 4+2=6 \text { caras } \\
& 4 \cdot 2=8 \text { vértices } \\
& 4 \cdot 3=12 \text { aristas }
\end{aligned}
$$

Por lo tanto, un cubo tiene 6 caras, 8 vértices y 12 aristas.
B Halla el número de caras, vértices y aristas de una pirámide cuadrada.

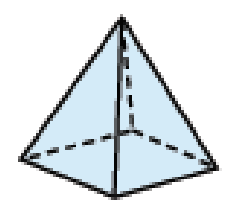

Una pirámide cuadrada tiene 4 lados en la base.

$$
\begin{aligned}
& 4+1=5 \text { caras } \\
& 4+1=5 \text { vértices } \\
& 4 \cdot 2=8 \text { aristas }
\end{aligned}
$$

Por lo tanto, una pirámide cuadrada tiene 5 caras, 5 vértices y 8 aristas.

Fuente: Andrews et al. (2014b, p. 87).

Finalmente, en la llustración 8 vemos un ejemplo del eje de Medida, en el que se trabaja la conversión de diferentes unidades de tiempos (semanas a días, días a horas y horas a minutos). En el contexto de un proyecto escolar sobre el cultivo de una planta, que durará 5 semanas, se pregunta cuántos días implica el desarrollo de este proyecto. Para ello se plantea la ecuación $(5 \cdot 7$ días $=$ días). 
Ilustración 8 - Ejemplo en el eje Medida.

\section{¿Cómo conviertes las unidades de tiempo?}

La clase está cultivando una planta a partir de una semilla. El proyecto durará 5 semanas.

¿Cuántos días hay en 5 semanas?

La imagen muestra cuánto

tiempo ha tardado la

semilla en germinar.

¿Cuántas horas es esto?

$$
\begin{gathered}
\text { Relación entre unidades de tiempo } \\
1 \text { semana }(\text { sem) }=7 \text { días } \\
1 \text { día }(\mathrm{d})=24 \text { horas } \\
1 \text { hora }(\mathrm{h})=60 \text { minutos }
\end{gathered}
$$

Como hay 7 días en una semana, el número de días en 5 semanas es $5 \cdot 7$.

$5 \cdot 7$ días $=$ días

$$
\frac{7}{35} \cdot 5
$$

$$
\begin{aligned}
& \text { Hacer una tabla para calcular la cantidad de } \\
& \text { horas en } 8 \text { días. }
\end{aligned}
$$

es$$
35 \text { días }=5 \text { semanas }
$$

\begin{tabular}{|l|l|c|c|c|c|c|c|c|c|}
\hline $\begin{array}{l}\text { Número } \\
\text { de días }\end{array}$ & 1 & 2 & 3 & 4 & 5 & 6 & 7 & 8 \\
\hline $\begin{array}{l}\text { Número } \\
\text { de horas }\end{array}$ & 24 & 48 & 72 & 96 & 120 & 144 & 168 & 192 \\
\hline
\end{tabular}

Hay 192 horas en 8 días.

Fuente: Charles et al. (2014, p. 218-219).

A modo de resumen, presentamos en la Tabla 4 la distribución de los ejemplos según el eje al que pertenecen las unidades en que se encuentran en los libros de texto. En ella vemos que estos se concentran en los ejes de Números y operaciones, y Patrones y álgebra, con el 96,8\% de los ejemplos encontrados sobre ecuaciones, y donde el primero de estos es más frecuente con un $56,5 \%$.

Observamos que en los dos primeros cursos solo se encuentran ejemplos en el eje de Números y operaciones y que a partir del tercero vemos en el eje de Patrones y álgebra. Esto va en concordancia con las directrices curriculares, que recién en tercero de Educación Primaria explicitan objetivos de aprendizajes relacionadas con este contenido matemático.

También, vemos que a partir del cuarto curso el eje en que predominan los ejemplos es en el de Patrones y álgebra, siendo el único en quinto y sexto. En los ejes de Medición y Geometría solo se ha encontrado un ejemplo en tercero y cuarto, respectivamente. Sin encontrar ejemplos en el eje de estadística (Datos y probabilidades). 
Tabla 4 - Distribución de la frecuencia $(F)$ y porcentaje $(P)$ de los ejemplos según eje temático

\begin{tabular}{|c|c|c|c|c|c|c|c|c|c|c|c|c|c|c|}
\hline \multirow{2}{*}{ Enunciado } & \multicolumn{2}{|c|}{$1^{0}$} & \multicolumn{2}{|c|}{$2^{\circ}$} & \multicolumn{2}{|c|}{$3^{\circ}$} & \multicolumn{2}{|c|}{$4^{\circ}$} & \multicolumn{2}{|c|}{$5^{\circ}$} & \multicolumn{2}{|c|}{$6^{\circ}$} & \multicolumn{2}{|c|}{ Total } \\
\hline & $\mathbf{F}$ & $\mathbf{P}$ & $\mathbf{F}$ & $\mathbf{P}$ & $\mathbf{F}$ & $\mathbf{P}$ & $\mathbf{F}$ & $\mathbf{P}$ & $\mathbf{F}$ & $\mathbf{P}$ & $\mathbf{F}$ & $\mathbf{P}$ & $\mathbf{F}$ & $\mathbf{P}$ \\
\hline Números y operaciones & 4 & 100 & 18 & 100 & 10 & 76,9 & 3 & 27,3 & 0 & 0 & 0 & 0 & 35 & 56,5 \\
\hline Patrones y álgebra & 0 & 0 & 0 & 0 & 2 & 15,4 & 7 & 63,6 & 7 & 100 & 9 & 100 & 25 & 40,3 \\
\hline Geometría & 0 & 0 & 0 & 0 & 0 & 0,0 & 1 & 9,1 & 0 & 0 & 0 & 0 & 1 & 1,6 \\
\hline Medición & 0 & 0 & 0 & 0 & 1 & 7,7 & 0 & 0 & 0 & 0 & 0 & 0 & 1 & 1,6 \\
\hline Total & 4 & 100 & 18 & 100 & 13 & 100 & 11 & 100 & 7 & 100 & 9 & 100 & 62 & 100 \\
\hline
\end{tabular}

\section{Conclusión}

Con este trabajo, por medio de las variables analizadas, hemos confirmado la importancia de las ecuaciones en la formación matemática de los escolares, reflejado por su presencia en las directrices curriculares y su implementación en los libros de texto.

Respecto al uso de ejemplos sobre ecuaciones, estos se observan a partir del tercer curso de Educación Primaria, basándose en la vivencia de los estudiantes de los cursos anteriores en los que se trabaja en mayor medida el concepto de igualdad, lo que hace referencia a lo establecido en el currículo.

El libro de texto en que se aprecia la mayor cantidad de ejemplos sobre ecuaciones es en segundo, pese a que en ese nivel no se explicita el trabajo con este objeto matemático en el currículo. Sobre las representaciones usadas en los ejemplos observamos que en su mayoría estos son una conjugación entre la pictóricas y simbólicas, es decir, que se presentan en conjunto. Esto puede estar dado por las orientaciones curriculares (MINEDUC, 2012), que establecen la progresión de lo concreto a lo pictórico (icónico) y a lo simbólico (abstracto) o en ambos sentidos.

Los enunciados conceptuales se observan con mayor frecuencia que los verbales. Los primeros están relacionados con la comprensión del lenguaje algebraico, se corrobora su importancia como aspecto fundamental del paso de la aritmética al álgebra en la matemática escolar, reconociendo esta simbología como propia del álgebra (HERNÁNDEZ; ANDONEGUí, 2003). Los enunciados conceptuales evidencian la unión de la enseñanza de la aritmética y el álgebra (LINS; GIMENEZ, 2001), considerando su resolución por medio de la comprensión del concepto de igualdad y sus propiedades (ROJAS, 2010).

El eje de Patrones y álgebra es declarado curricularmente como el eje que incorpora las ecuaciones. Sin embargo, la presencia de ejemplos se observa mayoritariamente en Números $y$ operaciones. Son escasos los ejemplos en los ejes de Geometría y Medida y nulos para el de Datos y probabilidades. Su presencia en otros ejes confirma su relevancia para el aprendizaje de diferentes temas, por ello es de extrañar que no se usen ejemplos sobre ecuaciones en las que se trabaje la media aritmética, en la que se puede dar este estadígrafo y se puede pedir el valor de 
uno de los datos.

A modo de sugerencia, en investigaciones futuras sobre ejemplos en los libros de texto, se podría ampliar la muestra de textos, niveles educativos (Enseñanza Secundaria) y contemplar otro tipo de variables.

\section{Referencias}

ALSINA, C. Mañana será otro día: un reto matemático llamado futuro In: GOÑI, J (Org.) EI currículum matemático en los inicios del siglo XXI. Barcelona: Graó, 2000. p. 13-21.

ANDREWS, A.; DIXON, J.; NORWOOD, K.; ROBY, T.; SCHEER, J.; BENNETT, J.; LUCKIE, L.; NEWMAN, V.; SCARCELLA, R.; WRIGHT, D. Matemática $4^{\circ}$ Básico. Santiago: Galileo, $2014 a$.

ANDREWS, A.; DIXON, J.; NORWOOD, K.; ROBY, T.; SCHEER, J.; BENNETT, J.; LUCKIE, L.; NEWMAN, V.; SCARCELLA, R.; WRIGHT, D. Matemática $5^{\circ}$ Básico. Santiago: Galileo, 2014b.

ANDREWS, A.; DIXON, J.; NORWOOD, K.; ROBY, T.; SCHEER, J.; BENNETT, J.; LUCKIE, L.; NEWMAN, V.; SCARCELLA, R.; WRIGHT, D. Matemática $6^{\circ}$ Básico. Santiago: Galileo, 2014c.

BERNAL, C. Problemas de ecuaciones de primer grado con una incógnita. 54 f. Trabajo Fin de Máster (Postgrado en Educación Especialidad Didáctica de las Matemáticas) - Universidad Autónoma de Barcelona, Barcelona, 2011.

BRAGA, G.; BELVER, J.L. El análisis de libros de texto: una estrategia metodológica en la formación de los profesionales de la educación. Revista Complutense de Educación, Madrid, v. 27, n. 1, p. 199-218, 2016.

CANTORAL, R.; MONTIEL, G.; REYES-GASPERINI, D. Análisis del discurso matemático escolar en los libros de texto, una mirada desde la Teoría Socioepestemológica. Avances de Investigación en Educación Matemática, Badajoz, v. 8, p. 9-28, 2015.

CÁRCAMO, M. Estudio sobre el uso de ejemplos en la enseñanza de la matemática: ecuaciones de primer grado. 68 f. Trabajo Fin de Máster (Máster Universitario en Investigación en Educación, Especialidad Educación Científica) - Universidad Autónoma de Barcelona, Barcelona, 2017.

CARRAHER, D. W. ; SCHLIEMANN, A. D.; BRIZUELA, B. M.; EARNEST, D. Arithmetic and algebra in early Mathematics Education. Journal for Research in Mathematics Education, Reston VA, v. 2, n. 37, p. 87-115, mar. 2006.

CASTRO, W. F.; MARTÍNEZ-ESCOBAR, J. D.; PINO-FAN, L. R. Niveles de algebrización de la actividad matemática escolar: Análisis de libros de texto y dificultades de los estudiantes.

REDIMAT. Journal of Research in Mathematics Education, Barcelona, v. 6, n. 2, p. 164-191, 2017.

CHARLES, R.; CALDWELL, J.; CAVANAGH, M.; CHANCELLOR, D.; COPLEY, J.; CROWN, W.; FENNELL, F.; RAMÍREZ, A.; SAMMONS, K.; SCHIELACK, J.; TATE, W.; VAN DE WALLE, J. Matemática $3^{\circ}$ Educación Básica. Santiago: Pearson, 2014.

CHEVALLARD, Y. La transposición didáctica. Del saber sabio al saber enseñado. Buenos Aires: Aique, 1991.

CORTÉS, C. Texto del Estudiante. Matemática 1 Básico. Santiago: Cal y Canto, 2017. 
DÍAZ-LEVICOY, D.; BATANERO, C.; ARTEAGA, P.; LÓPEZ-MARTíN, M.M. Análisis de los gráficos estadísticos presentados en libros de texto de Educación Primaria chilena. Educação Matemática Pesquisa, São Paulo, v. 17, n. 4, p. 715-739, 2015.

DÍAZ-LEVICOY, D.; ROA, R. Análisis de actividades sobre probabilidad en libros de texto para un curso de básica chilena. Revista Chilena de Educación Científica, Santiago, v. 13, n. 1, p. 9-19, 2014.

GODINO, J.; FONT, V. Razonamiento Algebraico y su Didáctica para maestros. Granada: Departamento de Didáctica de la Matemática de la Universidad de Granada, 2003.

GOLDENBERG, P.; MASON, J. Shedding light on and with example spaces. Educational Studies in Mathematics, Netherlands, v. 69, n. 2, p. 183-194, oct. 2008.

GÜEMES, R. Libros de texto y desarrollo del currículo en el aula. Un estudio de casos. 1994. 386 f. Tesis Doctoral (Doctorado en Educación) - Universidad de La Laguna, Tenerife, 1994.

HERBEL, B. A. From intended curriculum to written curriculum: examining the 'voice' of a mathematics textbook. Journal for Research in Mathematics Education, Reston VA, v. 38. n. 4, p. 344-369, 2007.

HERNÁNDEZ, M.; ANDONEGUÍ, M. Una experiencia didáctica referente a la introducción del tema ecuaciones en Educación Básica. In: DELGADO, J. (Org.) Acta Latinoamericana de Matemática Educativa. Santiago: CLAME, 2003, p. 176-182.

HERNÁNDEZ, R.; FERNÁNDEZ, C.; BAPTISTA, P. Metodología de la Investigación. México D.F.: McGraw Hill, 2006.

HO KHEONG, F.; RAMAKRISHNAN, C.; PUI WAH, B. L.; CHOO, M. Mi Matemática. Texto del Estudiante $2^{\circ}$. Santiago: Marshall Cavendish Education, 2017.

HURTADO, C. Análisis didáctico de las ecuaciones de primer grado con una incógnita y su impacto en la educación básica. In: CONGRESO IBEROAMERICANO DE EDUCACIÓN MATEMÁTICA, 7., 2013, Montevideo. Actas... Montevideo: SEMUR, 2013, p. 1045-1055.

JANVIER, C. Problems of Representation in the Teaching and Learning of Mathematics. Hillsdale, NJ: Lawrence Erlbaum Associates, 1987.

KIERAN, C.; FILLOY-YAGUE, E. El aprendizaje del álgebra escolar desde una perspectiva psicológica. Enseñanza de las Ciencias, Barcelona, v. 7, n. 3, p. 229-240, 1989.

LINS, R. C.; GIMENEZ, J. Perspectivas em aritmética e álgebra para o século XXI. Campinas: Papirus, 2001.

LÓPEZ-NOGUERO, F. El análisis de contenido como método de investigación. XXI. Revista de Educación, Huelva, v. 4, p. 167-180, 2002.

MINEDUC. Bases Curriculares. Educación Básica. Santiago: MINEDUC, 2012.

MINEDUC. Matemática. Programa de estudio tercer año básico. Santiago: MINEDUC, $2013 a$.

MINEDUC. Matemática. Programa de estudio cuarto año básico. Santiago: MINEDUC, 2013b.

MINEDUC. Matemática. Programa de estudio quinto año básico. Santiago: MINEDUC, 2013c.

MINEDUC. Matemática. Programa de estudio sexto año básico. Santiago: MINEDUC, 2013d. 
OLFOS, R. Lógica de justificación en la resolución de ecuaciones de primer grado. In: JORNADAS NACIONALES DE EDUCACIÓN MATEMÁTICA, 13., 2006, Viña del Mar. Actas... Viña del Mar: SOCHIEM, 2006.

ORTIZ, J. J. La probabilidad en los libros de texto. Granada: GEEUG, 2002.

OTERO, M. R.; ELICHIRIBEHETY, I.; ROA, M. El tratamiento dado a las ecuaciones en los textos ¿tiene en cuenta a los alumnos?. Educación Matemática, México DF, v. 12. n. 3, p. 18-29, 2000.

PÉREZ-SERRANO, G. Investigación cualitativa. Madrid: La Muralla, 1994.

ROJAS, P. Iniciación al álgebra escolar: elementos para el trabajo en el aula. In: ENCUENTRO COLOMBIANO DE MATEMÁTICA EDUCATIVA, 11., 2010, Bogotá. Memorias... Bogotá: Asociación Colombiana de Matemática Educativa, 2010. p. 115-131.

ROWLAND, T. The purpose, design and use of examples in the teaching of elementary mathematics. Educational Studies in Mathematics, Netherlands, v. 69, n. 2, p. 149-163, 2008.

SANTIBÁÑEZ, G.; DÍAZ-LEVICOY, D.; ROMO, C. Concepciones de los estudiantes que ingresan a la carrera de Arquitectura de la Universidad de Los Lagos sobre ecuaciones de primer grado. In: JORNADA DE MATEMÁTICA DE LA ZONA SUR, 23., 2009, Punta Arenas. Actas... Punta Arenas: Universidad de Magallanes, 2009.

SHIELD, M.; DOLE, S. Assessing the potential of mathematics textbooks to promote deep learning. Educational Studies in Mathematics, Netherlands, v. 82, n. 2, p. 183-199, 2013.

SILVA, E. T. Livro didático: do ritual de passagem à ultrapassagem. Em Aberto, Brasilia, v. 16, n. 69, p. 11-15, 1996.

VIDAL, R. El libro de texto de matemáticas en Chile en el último siglo 1910-2010. Cuadernos de Educación, Santiago, v. 27, p. 1-21, 2010.

WATSON, A.; CHICK, H. Qualities of examples in learning and teaching. ZDM Mathematics Education, Berlín, v. 43, n. 2, p. 283-294, 2010.

WATSON, A.; MASON, J. Mathematics as a constructive activity: learners generating examples. Mahwah, NJ: Lawrence Erlbaum, 2005.

ZODIK, I.; ZASLAVSKY, O. Characteristics of teachers' choice of examples in and for the mathematics classroom. Educational Studies in Mathematics, Netherlands, v. 69, n. 2, p. 165182, 2008. 